\title{
Survey of obstetric patients recollection of informed consent for regional anaesthesia
}

\section{J P O'Donoghue BSc (HONS) MBChB FRCA (ST7), T Keast (ST3), T Murphy (Cons)}

Anaesthetics, Queen Elizabeth University Hospital, Glasgow, UK

\section{Background and Aims:}

Informed consent has gained increasing importance in UK modern medicine since the landmark Montgomery case in 2015. The OAA last released an example guideline on regional analgesia and consent in 2010, this advised 'where time permits mothers should be given the epidural information sheets'.1 The GMC states patients should understand the information, retain the information, weigh up the information and communicate their wishes. At Queen Elizabeth University Hospital Glasgow, consent for urgent and emergency regional anaesthesia takes place in labour suite delivery rooms. Consent for elective regional anaesthesia takes place in labour suite recovery on the morning of surgery. This survey aimed to ascertain whether the consent process at our hospital allowed the patients to retain the information and risks they had given verbal consent for.

\section{Methods}

We conducted a survey asking women who had undergone regiona anaesthesia during their labour/delivery "What do you remember being told or reading about your epidural/spinal before it was done?". The consent discussion is recorded on our anaesthetic chart in the form of tick boxes. The same method was used to acknowledge patients recollection on the postnatal ward.

1. Please Record Consent/Risks discussed from Anaesthetic Chart:Technique

Failure/inadequate

Motor block

Nerve injury

Total Spinal

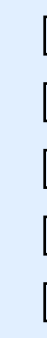

Info card read
$\downarrow$ BP
Headache
Infection
All the above

2. We also recorded what type of anaesthesia was used \& reason for it:-

E.g. Spinal / Epidural / CSE / Labour Analgesia /

Emergency theatre (Cat 1-2 LSCS/Trial) / Elective Theatre

(Cat 4 LSCS) / Urgent theatre (MROP, Cat 3 LSCS)

3. What do you remember being told or reading about your epidural/spinal before it was done?

[The question was delivered verbatim to the patient \& responses recorded as in question 1 (with the addition of tick boxes for pruritis and shivering)].

We tried to avoid any prompting and at most would say "Do you remember anything you were told about how the spinal/epidural would be done or the risks involved?". A minimal response on the details of technique was enough to gain a tick and in the same way details such as quoted incidence of e.g. headache were not required, just knowledge that a headache was a risk. We did not at any point ask specific questions e.g. "Do you remember being told about low blood pressure / nerve injury etc."

(All the women were consented for participation in the survey before question 3 was asked)

Caldicott Guardian Approval was obtained and ethical approval advice was sought for the project and it was deemed not to require review by an NHS Research Ethics Committee.

\section{Declaration}

A Similar Abstract on this work has been published:-

J P O'Donoghue, T Keast, T Murphy. Survey of obstetric patients recollection of informed consent for regional anaesthesia. International Journal of Obstetric Anesthesia Volume 35/S1, May 2018, S22 P.22.

And a poster was presented at:-

Obstetric Anaesthetists' Association, Annual Scientific Meeting, Belfast, 24-25 May 2018
Results

- 99 completed responses were received out of a total of 114 patients, 15 had either incomplete data or were not completed before the patient was discharged.

Our information card was read by 19 women out of 36 (52.8\%) who received an epidural and by 6 women out of $62(9.6 \%)$ who received a spinal.

Figure 1:- Number of topics that were recalled by the women out of the maximum 10 topics

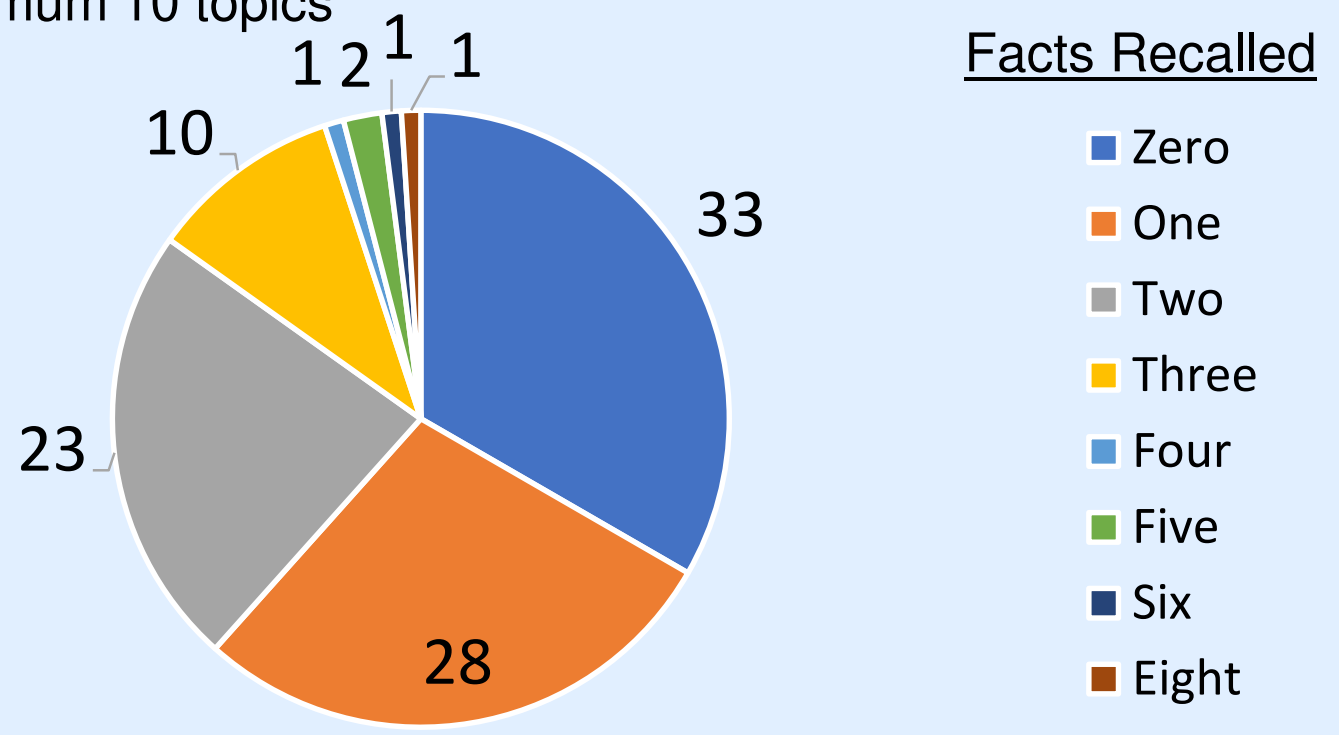

Figure 2:- Number of topics that were recalled by the women out of the maximum 10 topics dependant on the indication/urgency of regional anaesthesia required.

15

10
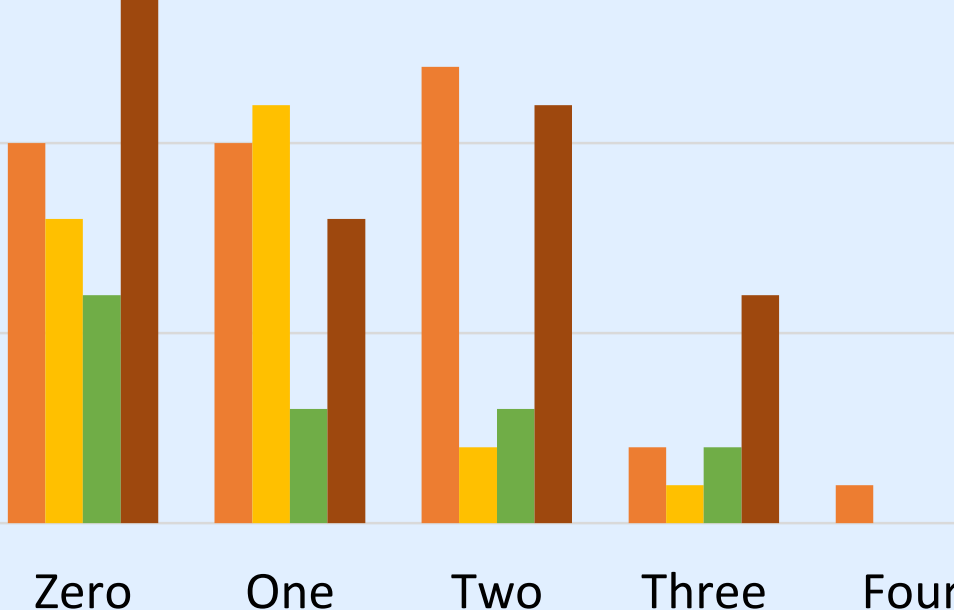

Labour

Elective Urgent

Emergency

Zero One Two Three Four Five Six Eight

Figure 3:- Number of women that recalled each of the individual 10 topics.

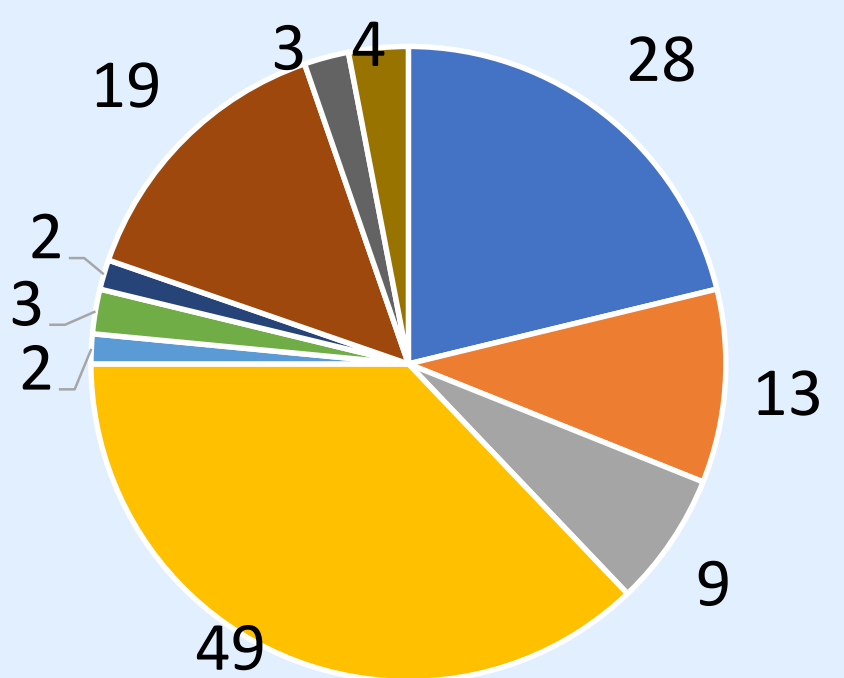

- Technique

- Failure/inadequate

Motor Block

Nerve Injury

Total Spinal

- Shivering

- Hypotension

- Headache

49

- The most recalled topics were nerve injury (49 women), technique (28 women) and headache (19 women). The topics least recalled total spinal and hypotension (both 2 women).

Discussion

From the results of our project it can be seen that more than $50 \%$ of women will retain only one fact and nearly $85 \%$ of women will retain only two facts from the consent process. There is little evidence to suggest that being consented for an elective caesarean section improves this retention of knowledge. Furthermore one of the rarest complications (nerve injury) is one of the most remembered, with one of the most common side effects (hypotension) being one of the least remembered.

Given that consent is obtained while the women are often in labour, emotionally stressed, in pain and had various drugs (Diamorphine, Entonox \& Oxytocin) it is not that surprising that patients are unable to adequately retain the information they are provided with. Our survey suggests that the current technique, timing and delivery of consent is unsatisfactory. It does not meet GMC or medico-legal standards, but more importantly it could be affecting our patients' ability to make informed choices regarding their care. 